NBER WORKING PAPER SERIES

MEASURING THE EFFECTS OF ADVERTISING:

THE DIGITAL FRONTIER

\author{
Randall Lewis \\ Justin M. Rao \\ David H. Reiley \\ Working Paper 19520 \\ http://www.nber.org/papers/w19520
}

\author{
NATIONAL BUREAU OF ECONOMIC RESEARCH \\ 1050 Massachusetts Avenue \\ Cambridge, MA 02138 \\ October 2013
}

\begin{abstract}
Much of this work was done when all the authors were at Yahoo! Research. We thank Garrett Johnson, Dan Nguyen, Sergiy Matusevych, Iwan Sakran, Taylor Schreiner, Valter Sciarillo, Christine Turner, Michael Schwarz, Preston McAfee, and numerous other colleagues for their assistance and support in carrying out the research. The views expressed herein are those of the authors and do not necessarily reflect the views of the National Bureau of Economic Research.
\end{abstract}

At least one co-author has disclosed a financial relationship of potential relevance for this research. Further information is available online at http://www.nber.org/papers/w19520.ack

NBER working papers are circulated for discussion and comment purposes. They have not been peerreviewed or been subject to the review by the NBER Board of Directors that accompanies official NBER publications.

(C) 2013 by Randall Lewis, Justin M. Rao, and David H. Reiley. All rights reserved. Short sections of text, not to exceed two paragraphs, may be quoted without explicit permission provided that full credit, including $\odot$ notice, is given to the source. 
Measuring the Effects of Advertising: The Digital Frontier

Randall Lewis, Justin M. Rao, and David H. Reiley

NBER Working Paper No. 19520

October 2013

JEL No. D47,L22,M37

\begin{abstract}
$\underline{\text { ABSTRACT }}$
Online advertising offers unprecedented opportunities for measurement. A host of new metrics, clicks being the leading example, have become widespread in advertising science. New data and experimentation platforms open the door for firms and researchers to measure true causal effects of advertising on a variety of consumer behaviors, such as purchases. We dissect the new metrics and methods currently used by industry researchers, attacking the question, "How hard is it to reliably measure advertising effectiveness?" We outline the questions that we think can be answered by current data and methods, those that we believe will be in play within five years, and those that we believe could not be answered with arbitrarily large and detailed data. We pay close attention to the advances in computational advertising that are not only increasing the impact of advertising, but also usefully shifting the focus from "who to hit" to "what do I get."
\end{abstract}

Randall Lewis

Google, Inc.

1600 Amphitheatre Parkway

Mountain View, CA 94043

ralewis@google.com

Justin M. Rao

Microsoft Research

641 Avenue of the Americas, 7th Floor

New York, NY 10011

justinra@microsoft.com
David H. Reiley

Google, Inc.

1600 Amphitheatre Parkway

Mountain View, CA 94043

reiley@google.com 


\title{
Measuring the Effects of Advertising: The Digital Frontier*
}

\author{
Randall Lewis \\ Google, Inc.
}

\author{
Justin M. Rao \\ Microsoft Research
}

\author{
David H. Reiley \\ Google, Inc.
}

\begin{abstract}
Online advertising offers unprecedented opportunities for measurement. A host of new metrics, clicks being the leading example, have become widespread in advertising science. New data and experimentation platforms open the door for firms and researchers to measure true causal effects of advertising on a variety of consumer behaviors, such as purchases. We dissect the new metrics and methods currently used by industry researchers, attacking the question, "How hard is it to reliably measure advertising effectiveness?" We outline the questions that we think can be answered by current data and methods, those that we believe will be in play within five years, and those that we believe could not be answered with arbitrarily large and detailed data. We pay close attention to the advances in computational advertising that are not only increasing the impact of advertising, but also usefully shifting the focus from "who to hit" to "what do I get."
\end{abstract}

\section{Introduction}

In the United States, advertising is a $\$ 200$ billion industry, annually. We all consume "free" services - those monetized by consumer attention to advertising - such as network television, email, social networking, and a vast array of online content. Yet despite representing a relatively stable $2 \%$ of GDP since World War I and paying for activities that comprise most of Americans' leisure time (American Time Use Survey, 2010), advertising remains poorly understood by economists. This is primarily because offline data have typically been insufficient for a firm (or researcher) to measure the true impact of advertising on consumer purchasing behavior. Theories of advertising that have important implications for competition are even harder to empirically validate. The digital era offers an unprecedented opportunity to bridge this informational divide. These advances, both realized and potential, can be attributed to two key factors: 1) individual-level data on ad delivery and purchases can be linked and made available to advertisers at low cost, and 2) ad delivery can be randomized at the individual level, generating exogenous variation essential to identifying causal effects. In this chapter we explore the dramatic improvement in the empirical measurements of the returns to advertising and also highlight fundamental challenges that remain.

\footnotetext{
${ }^{*}$ Much of this work was done when all the authors were at Yahoo! Research. We thank Garrett Johnson, Dan Nguyen, Sergiy Matusevych, Iwan Sakran, Taylor Schreiner, Valter Sciarillo, Christine Turner, Michael Schwarz, Preston McAfee, and numerous other colleagues for their assistance and support in carrying out the research.
} 
The ease of data collection in online advertising has led to standard reporting of precise quantitative data for advertising campaigns, most notably the click-through rate (CTR). Of course, the CTR of an ad is only an intermediate proxy for the real outcome of interest to the advertiser: ${ }^{1}$ increased purchases by consumers, both in the present and future. Despite these limitations, intermediate metrics such as the CTR have proved to be enormously useful dependent variables in automated targeting algorithms that match ads with consumers and contexts (Pandey and Olston, 2006; Gonen and Pavlov, 2007). A related intermediate metrics comes from "purchasing intent" surveys paired with randomized exposure to a firm's advertising. Cross-experiment analysis of such surveys has provided estimates of the relative value of targeted (vs. untargeted) advertising (Goldfarb and Tucker, 2011b), contextual relevance and ad intrusiveness (Goldfarb and Tucker, 2011a) and has informed the debate on privacy (Tucker, 2012).

The advances in both academic understanding and practical business practice attributable to these intermediate metrics should not be understated. But while general insights on how ad features impact users can guide advertising spend (and CTR based algorithms can make spending more efficient), a firm is presumably interested in measuring the overall returns on advertising investment: dollars sales casually linked to the campaign vs. dollars spent. Traditional econometric measurements of the returns to advertising typically rely on aggregate data fraught with endogeneity and identification problems (Bagwell, 2008). ${ }^{2}$ Despite the ability to run very large randomized control trials made possible by digital delivery and measurement, we have discovered a number of conceptual flaws in standard industry data collection and analysis methods used to measure the effects of advertising. In other words, the deluge of data on advertising exposures, clicks, and other associated outcomes have not necessarily created greater understanding of the basic causal effects of advertising, much less an understanding of more subtle questions such as the relative effectiveness of different types of consumer targeting, ad creatives, or frequency of exposure. The voluminous data, it seems to us, have not only created opportunity for intelligent algorithmic advances but also mistaken inference under the guise of "big data."

First, many models assume that if you do not click on the ad, then the ad has no effect on your behavior. Here we discuss work by coauthors Lewis and Reiley that showed online ads can drive offline sales, which are typically not measured in conversion or click rates; omitting these non-click-based sales leads to underestimating the total effects of advertising. Linking online and offline sales requires a dedicated experimental infractructure and third party data merging that have only recently become possible.

\footnotetext{
${ }^{1}$ Towards these ends, advertisers use browser cookies and click beacons to obtain a "conversion rate," the ratio of transactions attributed to the campaign to ad exposures. This measure seems ideal, but the attribution step is critical and current methods of assigning attribution have serious flaws, which we discuss in detail.

${ }^{2} \mathrm{~A}$ notable exception are the split cable TV experiments reported in Abraham et al. (1995) . The sample sizes in these experiments, run a in small U.S. town, were far smaller than online experiments and the authors did not report per experiment confidence intervals, rather they used cross-experiment techniques to understand what factors tended to influence consumers (for a follow-up analysis, see $\mathrm{Hu}$ et al., 2007 .
} 
Second, many models assume that if you do click on an ad and subsequently purchase, that conversion must have been due to that ad. This assumption seems particularly suspect in cases, such as search advertising, where the advertising is deliberately targeted at those consumers most likely to purchase the advertised product and temporally targeted to arrive when a consumer is performing a task related to the advertised good. Research has shown, for example, that a person searching for "ebay shoes" is very likely to purchase shoes on Ebay regardless of the intensity of advertising (Blake et al., 2013). While this is an extreme example, Blake et al. (2013) also show that the problem arises generally and measuring the degree to which advertising crowds out "organic conversions" is difficult to measure precisely. Näive approaches effectively assume this problem away, but since only "marginal clicks" are valuable and all clicks count towards the CTR, these methods will always overstate the quantity we really care about.

Third, more sophisticated models that do compare exposed to unexposed users to establish a baseline purchase rate typically rely on natural, endogenous advertising exposure and can easily generate biased estimates due to unobserved heterogeneity (Lewis et al., 2011). This occurs when the pseudo-control group does not capture important characteristics of the treated group, such as purchase intent or browsing intensity, which we show can easily be correlated with purchases whether advertising is present or not. Using data from 25 large experiments run at Yahoo! (Lewis and Rao, 2013), we have found that the standard deviation of purchases is typically ten times the mean. With such a noisy dependent variable, even a tiny amount of endogeneity can severely bias estimates. Beyond inducing bias in coefficient estimates, these specification errors also give rise to an over-precision problem. Because advertising typically explains only a very small fraction of the variance in consumer transaction behavior, even cleanly designed experiments typically require over a million subjects in order to be able to measure economically meaningful effects with any statistical precision (but even experiments with 1 million subjects can have surprisingly weak power, depending on the variance in sales).

Since experiments are generally considered the gold standard for precision ${ }^{3}$ (treatment is exogenous and independent across individuals), we should be suspicious if observational methods claim to offer higher precision. Further, with non-experimental methods, omitted heterogeneity or selection bias (so long as it can generate a partial R-squared of 0.00005 or greater) can induce bias that swamps plausible estimates of advertising effectiveness. Thus, if an advertiser does not use an experiment to evaluate advertising effectiveness, she has to have a level of confidence in her model that, frankly speaking, we find unreasonable given the obvious selection effects due to ad targeting and synchronization of advertising with product launches (ex. new iPad release) and demand shocks (such as the holiday shopping season).

Experimental work on measuring the dollar returns to advertising has given us a deeper appreciation for the limits of current data and methods. For example, we show that seemingly simple

\footnotetext{
${ }^{3}$ Not all experiments are created equal and methodologies to use pre-experiment data to enhance power as well as post-experiment trimming have advanced considerably in the digital era (Deng et al., 2013).
} 
"cross-channel" complementarity measures are exceedingly difficult to reliably estimate. Here we present evidence taken from Lewis and Nguyen (2013) that display advertising can increase keyword searches for the advertised brand. Some clicks on sponsored links are incorrectly attributed entirely to the search ad, but while the directional impact on searches can be documented we cannot tell if search ads perform better or worse in terms of the conversion rate when paired with display advertising. A similar experimental design at a much larger scale could answer this sort of question, but advertising to order 5-10 million individuals may be out of reach ${ }^{4}$ for most advertisers.

So while some questions are answerable with feasible (at least for some market participants) scale, we believe other questions are still outside the statistical power of current experimental infrastructure and methods. The primary example is the long-run effects of advertising. Essentially any analysis of the impact of advertising has to make a judgment call on which time periods to use in the analysis. Often this is the "campaign window" or the campaign window plus a chosen interval of time (typically 1-4 weeks). These thresholds almost certainly "wrong" because any impact that occurs after the cutoff should count in the return on investment (ROI) calculation. We explain why practitioners typically choose relatively short impact windows. The intuition is that the longer the time window under study, the lower the signal-to-noise ratio in the data (presuming the ad gets less impactful over time): point estimates of the cumulative effect tend to increase with longer time horizons, but standard errors of the effect increase by even more. This leads to an estimation "impossibility" analogous to the well-known "curse of dimensionality."

In the final two sections, we discuss how computational methods have increased advertising effectiveness through automated targeting and bidding. With automated targeting, the conversation is usefully shifted from "who to hit" to "what should I get." However, the key parameters of the automated system, such as the valuation of an action such as a click or conversion, the budget of the campaign and the duration, today must still be entered by a human. Indeed these are the exact parameters that we have argued are very difficult to estimate. We then discuss how new advances in ad-delivery, measurement and infrastructure are creating opportunities to advance the science of advertising. In the final section we present concluding remarks.

\section{Selection and power}

In today's dollars the average American is exposed to about $\$ 500$ worth of advertising per year. ${ }^{5}$ To break even, the universe of advertisers needs to net about $\$ 1.35$ in marginal profits per person per day. Given the gross margins of firms that advertise, our educated guess is that this roughly corresponds to about $\$ 4-6$ in incremental sales per day.

\footnotetext{
${ }^{4}$ Pun intended.

${ }^{5}$ Mean GDP per American is approximately $\$ 50,000$ in 2011, but median household income is also approximately $\$ 50,000$. The average household size is approximately 2.5 , implying an individual's share of median household income is roughly $\$ 20,000$. Thus, while $2 \%$ of GDP actually implies a per capita expenditure of $\$ 1,000$, we use $\$ 500$ as a round and conservative figure that is more representative of the average American's ad exposure.
} 
When an advertiser enters this fray, it must compete for consumers' attention. The cost per person of a typical campaign is quite low. Online "display" (banners, rectangular units, etc.) campaigns that deliver a few ads per day to a targeted individual cost about 1-2 cents per person per day. Television ads delivered once per person per day are only a bit more expensive. Note that even an aggressive campaign will typically only garner a small percentage of an individual's daily advertising exposure. We see many ads per day and presumably only a minority of them are relevant enough to a given person to impact his behavior.

The relatively modest average impact per person makes it difficult to assess cost-effectiveness. What complicates matters further is that individual-level sales are quite volatile for many advertisers. An extreme example is automobiles - the sales impact is either tens of thousands of dollars, or it is zero. ${ }^{6}$ While not as extreme, many other heavily advertised categories, including consumer electronics, clothing and apparel, jewelry, air travel, banking, and financial planning also have volatile consumption patterns. ${ }^{7}$ Exceptions to this class are single goods sold through direct conversion channels. Here we summarize work presented in Lewis and Rao (2013), which used 25 large advertising field experiments to quantify how individual expenditure volatility impacts the power of advertising effectiveness (hereafter, $a d f x$ ) experiments. In general, the signal-to-noise ratio is much lower than we typically encounter in economics.

We now introduce some formal notation to clarify the argument. Consider an outcome variable $y$ (sales), an indicator variable $x$ equal to 1 if the person was exposed to the advertising, and a regression estimate $\hat{\beta}$, which gives the average difference between the exposed (E) and unexposed (U) groups. In an experiment, exposure is exogenous - determined by a flip of the proverbial coin. In an observational study, one would also condition on covariates $W$, which could include individual fixed effects, and the following notation would use $y \mid W$. All the following results go through with the usual "conditional upon" caveat. We consider a regression of $y$ on $x$, whose coefficient $\hat{\beta}$ will give us a measure of the average dollar impact of the advertising per consumer.

We use standard notation for the sample means and variances of the sales of the exposed and unexposed groups, the difference in means between those groups, and the estimated standard error of that difference in means. We assume for simplicity that the exposed and unexposed samples are the same size $\left(N_{E}=N_{U}=N\right)$ as well as equal variances $\left(\sigma_{E}=\sigma_{U}=\sigma\right)$ to simplify the formulas:

$$
\begin{gathered}
\bar{y}_{E} \equiv \frac{1}{N_{E}} \sum_{i \in E} y_{i}, \bar{y}_{U} \equiv \frac{1}{N_{U}} \sum_{i \in U} y_{i} \\
\hat{\sigma}_{E}^{2} \equiv \frac{1}{N_{E}-1} \sum_{i \in E}\left(y_{i}-\bar{y}_{E}\right)^{2}, \hat{\sigma}_{U}^{2} \equiv \frac{1}{N_{U}-1} \sum_{i \in U}\left(y_{i}-\bar{y}_{U}\right)^{2} \\
\Delta \bar{y} \equiv \bar{y}_{E}-\bar{y}_{U}
\end{gathered}
$$

\footnotetext{
${ }^{6}$ The marginal profit impact is large, but clearly smaller, as it is the gross margin times the sales impact.

${ }^{7}$ For a bank, the consumption pattern once you sign up might be predictable, but the bank is making money from consumer switching which is "all or nothing."
} 


$$
\hat{\sigma}_{\Delta \bar{y}} \equiv \sqrt{\frac{\hat{\sigma}_{E}^{2}}{N_{E}}+\frac{\hat{\sigma}_{U}^{2}}{N_{U}}}=\sqrt{\frac{2}{N}} \cdot \hat{\sigma}
$$

We focus on two familiar econometric statistics. The first is the $R^{2}$ of the regression of $y$ on $x$, which gives the fraction of the variance in sales explained by the advertising (or, in the model with covariates, the partial $R^{2}$ after first partialling out covariates - for more explanation, see Lovell, 2008):

$$
R^{2}=\frac{\sum_{i \in U}\left(\bar{y}_{U}-\bar{y}\right)^{2}+\sum_{i \in E}\left(\bar{y}_{E}-\bar{y}\right)^{2}}{\sum_{i}\left(y_{i}-\bar{y}\right)^{2}}=\frac{2 N\left(\frac{1}{2} \Delta \bar{y}\right)^{2}}{2 N \hat{\sigma}^{2}}=\frac{1}{4}\left(\frac{\Delta \bar{y}}{\hat{\sigma}}\right)^{2}
$$

Second is the $t$-statistic for testing the hypothesis that the advertising had no impact:

$$
t_{\Delta \bar{y}}=\frac{\Delta \bar{y}}{\hat{\sigma}_{\Delta \bar{y}}}=\sqrt{\frac{N}{2}}\left(\frac{\Delta \bar{y}}{\hat{\sigma}}\right)
$$

In both cases, we have related a standard regression statistic to the ratio between the average impact on sales and the standard deviation of sales between consumers.

In the following hypothetical example, we calibrate values using approximately median values from 19 retail sales experiments run at Yahoo!. For expositional ease, we will discuss it as if it is a single experiment. The campaign goal is a $5 \%$ increase in sales during the two weeks of the campaign, which we will use as our "impact period" of interest. During this period, customers of this advertiser make purchases with a mean of $\$ 7$ and a standard deviation of $\$ 75 .{ }^{8}$ The campaign costs $\$ 0.14$ per customer, which amounts to delivering $20-100$ display ads at a price of $\$ 1-\$ 5 \mathrm{CPM},{ }^{9}$ and the gross margin (markup over cost of goods sold, as a fraction of price) is assumed to be about $50 \% .{ }^{10}$ A $5 \%$ increase in sales equals $\$ 0.35$ per person, netting profits of $\$ 0.175$ per person. Hence, the goal for this campaign is to deliver a $25 \%$ return on investment (ROI): $\$ 0.175 / \$ 0.14=1.25 .^{11}$

The estimation challenge facing the advertiser in this example is to detect a $\$ 0.35$ difference in sales between the treatment and control groups amid the noise of a $\$ 75$ standard deviation in sales. The ratio is very low: 0.0047 . From our derivation above, this implies an $R^{2}$ of:

$$
R^{2}=\frac{1}{4} \cdot\left(\frac{\$ 0.35}{\$ 75}\right)^{2}=0.0000054
$$

That is, even for a successful campaign with a relatively large ROI, we expect an $R^{2}$ of only 0.0000054. This will require a very large $N$ to identify any influence at all of the advertising, let

\footnotetext{
${ }^{8}$ Based on data-sharing arrangements between Yahoo! and a number of advertisers spanning the range from discount to high-end retailers, the standard deviation of sales is typically about 10 times the mean. Customers purchase goods relatively infrequently, but when they do, the purchases tend to be quite large relative to the mean.

${ }^{9} \mathrm{CPM}$ is the standard for impression-based pricing for online display advertising. It stands for "cost per mille" or "cost per thousand;" $\mathrm{M}$ is the roman numeral for 1,000 .

${ }^{10}$ We base this assumption on our conversations with retailers and our knowledge of the industry.

${ }^{11}$ For calibration purposes, note that if the gross margin were $40 \%$ instead of $50 \%$, this would imply a $0 \%$ ROI.
} 
alone give a precise confidence interval. Suppose we had 2 million unique users evenly split between test and control in a fully randomized experiment. With a true ROI of $25 \%$ and a ratio of 0.0047 between impact size and standard deviation of sales, the expected $t$-stat is 3.30 , using the above formula. This corresponds to a test with power of about $95 \%$ at the $10 \%$ ( $5 \%$ one-sided) significance level, as the normally distributed t-stat should be less than the critical value of 1.65 about $5 \%$ of the time given the true effect is a 25\% ROI. With 200,000 unique customers, the expected t-stat is 1.04, indicating the test is hopelessly underpowered to reliably detect an economically relevant impact: under the alternative hypothesis of a healthy $25 \%$ ROI, we fail to reject the null $74 \%$ of the time. ${ }^{12}$

The low $R^{2}=0.0000054$ for the treatment variable $x$ in our hypothetical randomized trial has serious implications for observational studies, such as regression with controls, difference-indifferences, and propensity score matching. A very small amount of endogeneity would severely bias estimates of advertising effectiveness. An omitted variable, misspecified functional form, or slight amount of correlation between browsing behavior and sales behavior generating $R^{2}$ on the order of 0.0001 is a full order of magnitude larger than the true treatment effect. Compare this to a classic economic example such as the Mincer wage/schooling regression (Mincer, 1962), in which the endogeneity is roughly $1 / 8$ the treatment effect (Card, 1999). For observational studies, it is always important to ask, "What is the partial $R^{2}$ of the treatment variable?" If it is very small, as in the case of advertising effectiveness, clean identification becomes paramount, as a small amount of bias can easily translate into an economically large impact on the coefficient estimates.

Our view has not yet been widely adopted, however, as evidenced by the following quotation from the president of comScore, a large data-provider for online advertising:

Measuring the online sales impact of an online ad or a paid-search campaign - in which a company pays to have its link appear at the top of a page of search results - is straightforward: We determine who has viewed the ad, then compare online purchases made by those who have and those who have not seen it.

\section{Abraham, 2008. Harvard Business Review}

The argument we have made shows that simply comparing exposed to unexposed can lead to bias that is many orders of magnitude larger than the true size of the effect. Indeed, this methodology led the author to report as much as a 300\% improvement in outcomes for the exposed group, which seems surprisingly high (it would imply, for instance, that advertisers are grossly underadvertising). Since all ads have some form of targeting, ${ }^{13}$ endogeneity is always a concern. For example, most

\footnotetext{
${ }^{12}$ Note that when a low powered test does, in fact, correctly reject the null, the point estimates conditional on rejecting will be significantly larger than the alternatively hypothesized ROI.

13 "Untargeted" advertising usually has implicit audience targeting based on where the ads are shown or implicit complementary targeting due to other advertisers purchasing targeted inventory and leaving the remnant inventory to be claimed by advertisers purchasing "untargeted" advertising inventory.
} 
display advertising aims to reach people likely to be interested in the advertised product, where such interest is inferred using demographics or past online behavior of that consumer. Similarly, search advertising targets consumers who express interest in a good at a particular point in time, where the interest is inferred from their search query (and potentially past browsing behavior). In these cases, comparing exposed to unexposed is precisely the wrong thing to do. By creating exogenous exposure, the first generation of advertising experiments have been a step in the right direction. Experiments are ideal-necessary, in fact-for solid identification.

Unfortunately, for many advertised products the volatility of sales means that even experiments with millions of unique users can still be underpowered to answer basic questions such as "Can we reject the null hypothesis that the campaign had zero influence on consumer behavior?" Measuring sales impact, even in the short-run, turns out to be much more difficult than one might have thought. The ability to randomize ad delivery on an individual level and link it to data on customer-level purchasing behavior has opened up new doors in measuring advertising effectiveness, but the task is still by no means easy. In the remainder of the paper we discuss these challenges. The next section focuses on using the right metrics to evaluate advertising.

\section{The evolution of advertising metrics}

The click-through-rate, or CTR, has become ubiquitous in the analysis and decision-making surrounding online advertising. It is easy to understand why: clicks are cleanly defined, easily measurable, and occur relatively frequently. An obvious but intuitively appealing characteristic is that an ad-click cannot occur in the absence of an ad. If one runs 100,000 ads and gets a $0.2 \%$ CTR (a typical rate for a display ad or a low-ranked search ad), it is tempting to conclude the ad caused 200 new website visits. The assumption may well be true for new or little-known brands. But for well-known advertisers, there are important ways that consumers might navigate to the site in the absence of an ad, such as browsing directly to the site by typing the name in the URL window of the browser or finding it in organic (that is, not paid or "sponsored") search results on a topic like "car rental." It is a mistake to assume that all of those 200 visits would not have occurred in the absence of the ad - that is, those clicks may be crowding out visits that would have happened via other means (Kumar and Yildiz, 2011; Chan et al., 2010).

The overcounting problem is surmountable with randomized trials where the control group is used to estimate the "baseline arrival rate." For example, a sponsored search ad could be turned off during random times of the day and the firm could measure arrivals from the search engine for when the ad is running and when it is not (this approach is used in Blake et al., 2013). ${ }^{14}$ A deeper problem with the CTR is what it misses. First, it does little for "brand advertisers" - firms that are not trying to generate immediate online sales, but rather to promote awareness and good-will

\footnotetext{
${ }^{14}$ Despite the simplicity of their design, Blake et al. estimate that their employer, eBay, had been wasting tens of millions of dollars a year.
} 
for the brand. To assess their spend, brand advertisers have traditionally relied on surveys that attempt to measure whether a campaign raised the opinion of the firm in the minds of their target consumers (Goldfarb and Tucker, 2011b). Linking the surveys to future purchasing behavior adds another layer of complexity, both because the time frame from exposure to sale is longer (something we will discuss in more detail in Section 5) and because it requires a reliable link from hypothetical responses to actual behavior, which can be fraught with what is known as "hypothetical bias" (Dickie et al., 1987; Murphy et al., 2005). One common approach to neutralize hypothetical bias is to use the surveys primarily for relative comparison of advertising campaigns.

For advertisers that sell goods both online and in brick-and-mortar stores the click (or online conversions) can be a poor proxy for overall ROI. Lewis and Reiley (2013b) show that for a major retailer, the majority of the sales impact comes offline. Johnson, Lewis, and Reiley (2013) link the offline impact to consumers who lived in close physical proximity to one of the retailer's locations. These studies indicate purely online measurements can induce a large negative bias in measuring the returns to advertising. For firms that do business on and offline it is essentialy to develop the infrastructure to link online ad exposure to offline sales.

The click is an intermediate metric for short-term sales (and short-term sales might be considered a further downstream metric for the net-present-discounted value of a customer). Alternatively, however, advertisers can now run "cost per acquisition" (CPA) advertising on many ad exchanges. ${ }^{15}$ An acquisition, or conversion, is defined as a successful transaction that has a "qualifying connection" to the advertisement. On the surface, focusing on conversions seems more attractive than clicks because it is a step closer to sales. Unfortunately this benefit brings with it what is known as the "attribution problem:" which ad gets "credit" for a given sale? Suppose a consumer views and clicks a given ad, but does not purchase on the same day. Over the next few days, she sees a host of other ads for the product (which is likely, given a practice known as "re-targeting") and then purchases the good. Which ad should get credit for the purchase?

Ad exchanges tend to use a set of rules to solve these problems from an accounting perspective. Common rules include requiring a click for credit or only counting the "last click" (so if a consumer clicks a re-targeted ad, that ad gets credit). Requiring a click seems to make sense and is enormously practical as it means a record of all viewers that see the ad but do not click need not be saved. ${ }^{16}$ However, requiring a click errs in assuming that ads can only have an impact through clicks, which is empirically not true (Lewis et al., 2012). The "last click" rule also has intuitive appeal. The reasoning goes as follows: had the last click not occurred, the sale would not have happened. Even if this were true, which we doubt, the first click or ad view might have led to web search, or other activity, including the behavioral markers used for re-targeting, which made the last click possible. The causal attribution problem is typically "solved" by ad-hoc rules set by the ad exchange or

\footnotetext{
${ }^{15}$ But not the major search engines, as of August 2013.

${ }^{16} \mathrm{~A}$ CTR of $\approx 0.2 \%$, meaning, storage and processing costs of only clicks involves only $\frac{1}{500}$ of the total ad exposure logs.
} 
publisher, such as "the first ad and the last ad viewed before purchase each get $40 \%$ of the credit, while the intermediate ad views share the remaining $20 \%$ of the credit for the purchase." 17 A proliferation of such rules gives practitioners lots of choices, but none of them necessarily gives an unbiased measurement of the performance of their ad spending. In the end, such complicated payment rules might make the click more attractive after all.

The attribution problem is also present in the question of complementaries between display and search advertising. Recent work has shown that display ads causally influence search behavior (Lewis and Nguyen, 2013). The authors demonstrate this by comparing the search behavior of users exposed to the campaign ad to users who would have been served the campaign ad but were randomly served a placebo. Brand-related keywords were significantly more prevalent in the treatment group as compared to the control. The attribution problem has received more attention in online advertising because of the popularity of cost-per-acquisition and cost-per-click payment mechanisms, but it applies to offline settings as well. How do we know, for example, whether an online ad was more responsible for an online conversion than was the television ad that same user saw? Nearly every online campaign occurs contemporaneously with a firm's offline adverising through media such as billboards and television because large advertisers are continuously advertising across many media. ${ }^{18}$ Directly modeling the full matrix of first-order interactions is well beyond the current state of the art. Indeed in every paper we know of evaluating online advertising, the interactions with offline spending is ignored.

Our discussion thus far has indicated that the evolution of advertising metrics has brought forth new challenges linking these metrics to the causal impact on sales. However one way in which intermediate metrics have proved unambiguously useful for advertisers is providing relatively quick feedback on targeting strategies allowing for algorithmic adjustments to the ad serving plan. For instance, one is not likely to be comfortable assuming that the click captures all relevant effects of the ad, but might be comfortable assuming that a higher CTR is always better than a lower CTR (this may be true for a class of advertisements a firm uses, for instance), then bandit algorithms can be applied to improve the efficiency of advertising spend and give relative comparisons of campaign effectiveness, allowing on to prioritize better performing advertisements (Pandey and Olston, 2006; Gonen and Pavlov, 2007). We discuss these advances in more detail in Section 7.

\section{A case study of a large-scale advertising experiment}

To get a better idea of how large advertising experiments are actually run, in this section we present a case study taken from Lewis and Reiley (2013b) (herein "LR"). LR ran a large-scale experiment for a major North American retailer. The advance the paper makes is linking existing customers

\footnotetext{
${ }^{17}$ Source: https://support.google.com/analytics/bin/answer.py?hl=en\&answer=1665189

${ }^{18}$ (Lewis and Reiley, 2013a) show that Super Bowl commercials cause viewers to search for brand-related content across a wide spectrum of advertisers.
} 
in the retailer's sales records, for both online and brick-and-mortar sales, to a unique online user identifier, in this case the customer's Yahoo! username.

The experiment was conducted as follows. The match yielded a sample of 1,577,256 individuals who matched on name and either email or postal address. The campaign was targeted only to existing customers of the retailers as determined by the match. Of these matched users, LR assigned $81 \%$ to a treatment group who subsequently viewed two advertising campaigns promoting the retailer when logged into Yahoo's services. The remaining $19 \%$ were assigned to the control group and prevented from seeing any of the retailer's ads from this campaign on the Yahoo! network of sites. The simple randomization was designed to make the treatment-control assignment independent of all other relevant variables.

Table 1: Summary Statistics for the Campaigns

\begin{tabular}{lrrr}
\hline & Campaign 1 & Campaign 2 & Both Campaigns \\
\hline Time Period Covered & Early Fall '07 & Late Fall '07 & \\
Length of Campaign & 14 days & 10 days & \\
Number of Ads Displayed & $32,272,816$ & $9,664,332$ & $41,937,148$ \\
Number of Users Shown Ads & 814,052 & 721,378 & 867,839 \\
$\%$ Treatment Group Viewing Ads & $63.7 \%$ & $56.5 \%$ & $67.9 \%$ \\
Mean Ad Views per Viewer & 39.6 & 13.4 & 48.3 \\
\hline
\end{tabular}

Source: Lewis and Reiley (2013b).

The treatment group of 1.3 million Yahoo! users was exposed to two different advertising campaigns over the course of two months in fall 2007, separated by approximately one month. Table 1 gives summary statistics for the campaigns, which delivered 32 million and 10 million impressions, respectively. The two campaigns exposed ads to a total of 868,000 users in the 1.3million-person treatment group. These individuals viewed an average of 48 ad impressions per person.

The experiment indicated an increase in sales of nearly $5 \%$ relative to the control group during the campaign, a point estimate which would translate to an extremely profitable campaign (with the retailer receiving nearly a $100 \%$ rate of return on the advertising spending). However, purchases had sufficiently high variance (due in part to $95 \%$ of consumers making zero purchases in a given week) to render the point estimate not statistically significantly different from zero at the $5 \%$ level. Controlling for available covariates (age, gender, state of residence) did not meaningfully reduce standard errors. This is good example of how economically important effects of advertising can be statistically very difficult to detect, even with a million-person sample size. Just as we saw in Section 2, we see here that the effects of advertising are so diffuse, explaining such a small fraction of the overall variance in sales, that the statistical power can be quite low. For this experiment, power calculations show that assuming the alternative hypothesis that the ad broke even is true, the probability of rejecting the null hypothesis of zero effect of advertising is only $21 \%$. 
The second important result of this initial study was a demonstration of the biases inherent in using cross-sectional econometric techniques when there is endogenous advertising exposure. This is important because these techniques are often employed by quantitative marketing experts in industry. Abraham (2008), for example, advocates comparing the purchases of exposed users to unexposed users, despite the fact that this exposure is endogenously determined by user characteristics and browsing behavior, which might easily be correlated with shopping behavior. To expose the biases in these methods, LR temporarily "discarded" their control group and compared the levels of purchases between exposed and (endogenously) unexposed parts of the treatment group. The estimated effects of advertising were three times as large as in the experiment, and with the opposite sign! This erroneous result would also have been deemed highly statistically significant. The consumers who browsed Yahoo! more intensely during this time period (and hence were more likely to see ads) tended to buy less, on average, at the retailer, regardless of whether they saw the ads or not (this makes sense, because as we will see most of the ad effect occured offline). The control group's baseline purchases prior to the ad campaign showed the same pattern. Without an experiment an analyst would have had no way of realizing the extent of the endogeneity bias (in this case, four times as large as the true causal effect size) and may have come to a strikingly wrong conclusion.

Observing the consistent differences between exposed and unexposed groups over time motivated LR to employ a difference-in-differences estimator. Assuming that any unobserved heterogeneity was constant over time allowed LR to take advantage of both exogenous and endogenous sources of variation in advertising exposure, which turned out to reduce standard errors to the point where the effects were statistically significant at the $5 \%$ level. The point estimate was approximately the same as (though slightly higher than) the straight experimental estimate, providing a nice specification check. With this estimator, LR also demonstrated that the effects of the advertising were persistent for weeks after the end of the campaign, that the effects were significant for in-store as well as online sales (with $93 \%$ of the effect occurring offline), and that the effects were significant even for those consumers who merely viewed but never clicked the online ads (with an estimated $78 \%$ of the effect coming from non-clicking viewers). In a companion paper (Lewis and Reiley, 2012), the authors also showed that the effects were particularly strong for the older consumers in the sample - sufficiently strong to be statistically significant even with the simple (less efficient) experimental estimator.

In a follow-up study, Johnson, Lewis, and Reiley (2013, henceforth JLR) improved on some of the weaknesses of the design of the original LR experiment. First, JLR ran "control ads" (advertising one of Yahoo's own services) to the control group, allowing them to record which control-group members would have been exposed to the ad campaign if they had been in the treatment group. This allowed them to exclude from their analysis those users (in both treatment and control groups) who were not exposed to the ads and therefore contributed noise but no signal to the statistics. Second, 
JLR convinced the advertiser to run equal-sized treatment and control groups, which improved statistical power relative to the LR article's 81:19 split. Third, JLR obtained more detailed data on purchases: two years of pre-campaign sales data on each individual helped to explain some of the variance in purchases, and disaggregated daily data during the campaign allowed them to exclude any purchases that took place before the first ad delivery to a given customer (which therefore could not have been caused by the ads, so including those purchases merely contributed noise to the estimates). The more precise estimates in this study corroborate the results of LR, showing point estimates of a profitable $5 \%$ increase in advertising which are statistically significant at the $5 \%$ level, though the confidence intervals remain quite wide.

\section{Activity bias}

In the preceding sections, we have presented this argument on an abstract level, arguing that the since the partial $R^{2}$ of advertising, even for a successful campaign, is so low (on the order of 0.00001 or less), the likelihood of omitted factors not accounting for this much variation is unlikely, especially since ads are targeted across time and people. In this section we show that our argument is not just theoretical. Here identify a bias that we believe is present in most online ad serving; in past work we gave it the name "activity bias" (Lewis et al., 2011). "Activity bias" is a form of selection bias based on the following two features of online consumer behavior: 1) since one has to be browsing online to see ads, those browsing more actively on a given day are more likely to see your ad, and 2) active browsers tend to do more of everything online, including buying goods, clicking links and signing up for services. any of the selection mechanisms that lead to their exposure to the advertising are highly correlated with other online activities. Indeed many of the selection mechanisms that lead to their exposure to the advertisin, such as retargeting ${ }^{19}$ and behavioral targeting, are highly correlated with other online activities. Hence, we see that ad exposure is highly and non-causally correlated with many online activities, making most panel and time-series methods subject to bias. In a non-experimental study, the unexposed group, as compared to the group exposed to an ad, typically failed to see the ad for one or both of the following reasons: the unexposed users browsed less actively or the user did not qualify for the targeting of the campaign. When the former fails, we have activity bias. When the latter fails, we have classic selection bias.

In our 2011 paper, we explored three empirical examples demonstrating the importance of activity bias in different types of web browsing. The first application investigates the causal effects of display ads on users' search queries. In Figure 5 we plot the time series of the number of searches by exposed users for a set of keywords deemed to be brand-relevant for a firm. The figure shows results for a time period that includes a one-day display-advertising campaign for a national brand on www.yahoo.com.

\footnotetext{
${ }^{19}$ For a discussion and empirical analysis of retargeting see Lambrecht and Tucker (2011).
} 


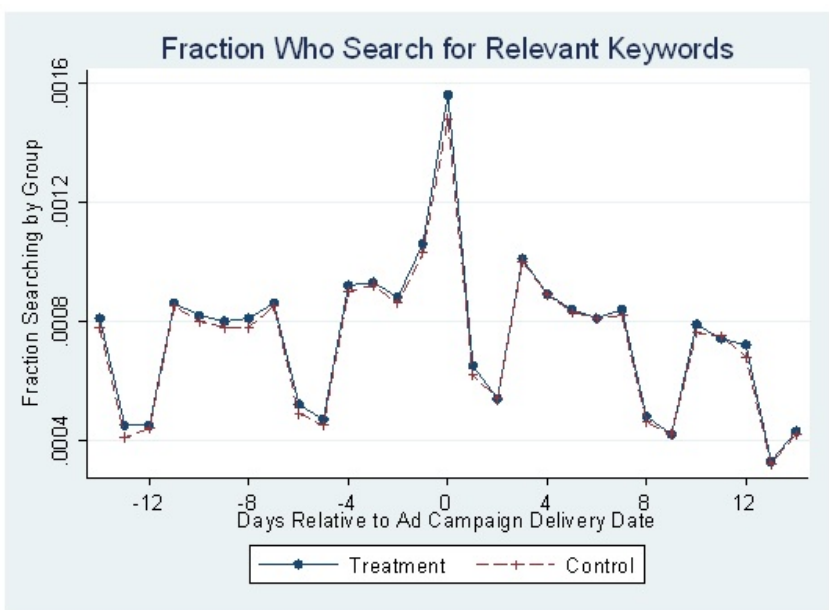

Figure 1: Brand keyword search patterns over time. Source: Lewis, Rao and Reiley (2011)

The campaign excluded a randomized experimental control group, though for the moment we ignore the control group and focus on the sort of observational data typically available to advertisers (the treatment group, those that saw the firm's advertisements). The $\mathrm{x}$-axis displays days relative to the campaign date, which is labeled as Day 0. One can easily see that on the date of the ad, ad viewers were much more likely to conduct a brand-relevant search than on days prior or following. The advertising appears to double baseline search volume. Is this evidence of a wildly successful ad? Actually, no. Examining the control group, we see almost the same trend. Brand-relevant keyword searches spike for even those who saw a totally irrelevant ad. What is going on? The control group is, by design of the experiment just as active online as the treatment group, searching for more of everything, not just the brand-relevant keywords of interest. The time series also shows that search volume is positively serially correlated over time and shows striking day of week effects - both could hinder observational methods. The true treatment-control difference is a statistically significant, but far more modest, $5.1 \%$. Without an experiment, we would have no way of knowing the baseline "activity-related increase" which we infer from the control group. Indeed, we might have been tempted to conclude the ad was wildly successful.

Our second application involves correlation of activity not just across a publisher and search engine, but across very different domains. We ran a marketing study to evaluate the effectiveness of a video advertisement promoting the Yahoo! network of sites. We recruited subjects on Amazon Mechanical Turk, showed them the video and gave them a Yahoo! cookie so we could track their future behavior. Using the cookie we could see if the ad really generated more Yahoo! activity. The control group saw a political ad totally unrelated to Yahoo! products and services. Again, we ignore the control group to begin. Figure 5 has the same format as Figure 5 Day 0 on the x-axis labels the day an individual saw the video ad (with the actual calendar date depending on the day the subject participated in the study). 

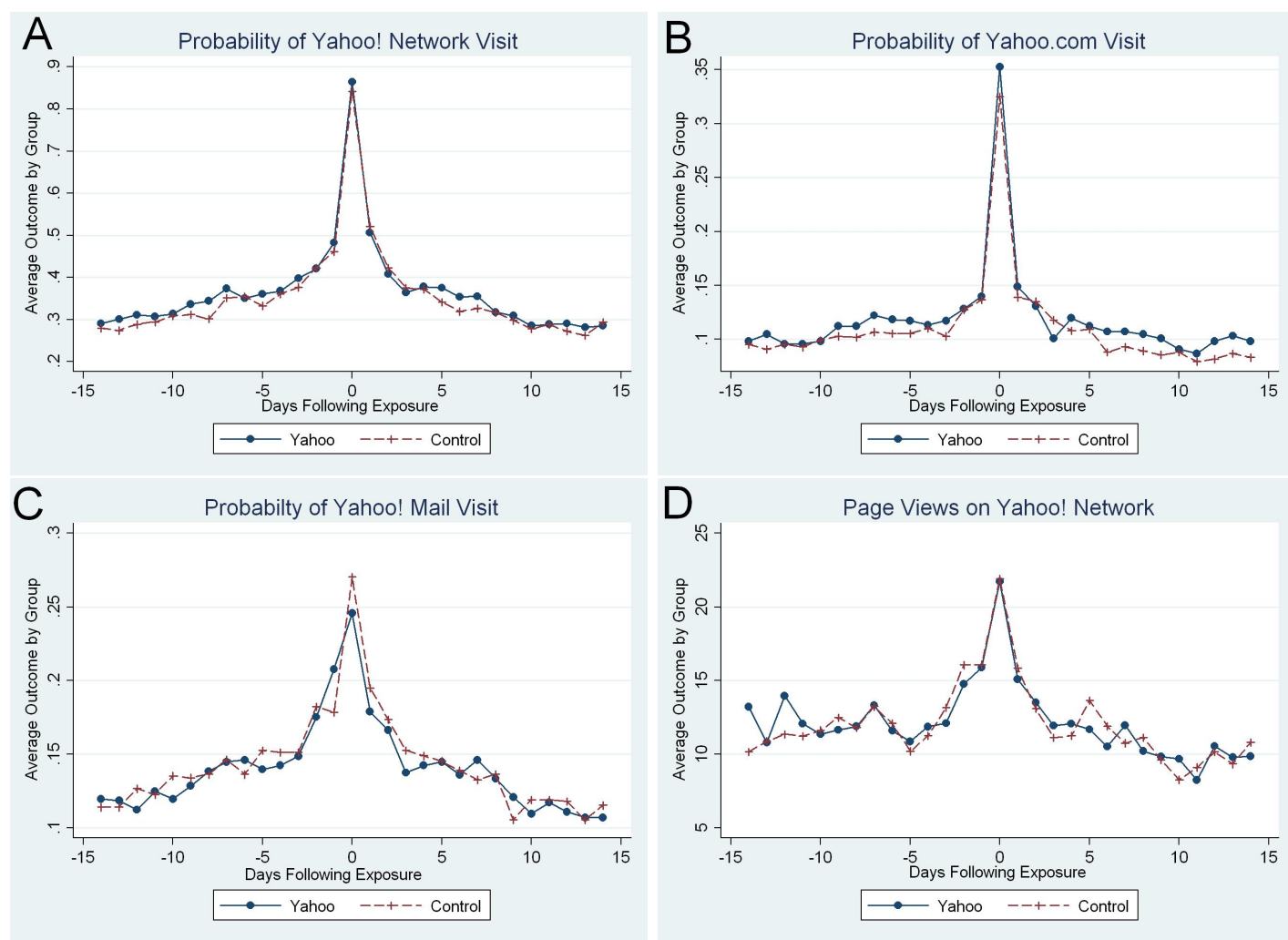

Figure 2: The effect on various Yahoo! usage metric of exposure to treatment/control ads. Panels A-C: Probability of at least 1 visit to the Yahoo! network, Yahoo.com and Mail respectively. Panel D: Total page views on the Yahoo! network. Source: Lewis, Rao and Reiley (2011)

Examining the treatment group, we can see that on the day of and the days following adexposure, subjects were much more likely to visit a Yahoo! site as compared to their baseline propensity, indicating a large apparent lift in engagement. However, data on the control group reveals the magnitude of activity bias - a very similar spike in activity on Yahoo! occurs on the day of placebo exposure as well. Both groups also show some evidence of positive serial correlation in browsing across days: being active today makes it more likely that you will be active tomorrow as compared to several days from now. People evidently do not engage in the same online activities (such as visiting Yahoo! and visiting Amazon Mechanical Turk) every day, but they engage in somewhat bursty activity that is contemporaneously correlated across sites. Online activity leads to ad exposure, which mechanically tends to occur on the same days as outcome measures we hope to affect with advertising. In the absence of a control group, we can easily make errors in causal inference due to activity bias. In this particular case, the true causal effect of the ad was estimated to be small and not statistically significant - given the cost of running a video ad, it was probably not worth showing, but the biased estimates would have led us to a wrong conclusion in this regard.

The third application again involves multiple websites. This time the outcome measure was 
filling out a new-account sign-up form at an online brokerage advertised on Yahoo! Finance. Again our results show that even those who were randomly selected to see irrelevant placebo ads were much more likely to sign up on the day they saw the (placebo) ad than on some other day. We refer the reader to our original paper for the details, stating here that the results are very similar to the ones we have just presented (the now familiar mountain-shaped graphs are again present). With activity bias it seems that one could erroneously "show" that nearly any browsing behavior is caused by nearly any other browsing behavior! We hope that our results will cause industry researchers to be more cautious in their conclusions. Activity bias is a real form of bias that limits the reliability of observational methods.

In the absence of an experiment, researchers may be able to use some other cross-validation technique in order to check the robustness of causal effects. For example, one could measure the "effect" of movie advertisements on searches for the seemingly irrelevant query "car rental." Similarly, one could check whether (placebo) ad views of a Toyota ad on the New York Times website on May 29 "causes" the same effect on Netflix subscriptions that day as did the actual Netflix ad on the New York Times website on May 30. Differences in differences using such pseudocontrol groups will likely give better estimates of true causal effects than simple time-series or cross-sectional studies, though of course a randomized experiment is superior if it is available (Lewis et al., 2011). ${ }^{20}$

Is activity bias a new phenomenon that is unique to the online domain? While it is not obvious that offline behavior is as bursty and as contemporaneously correlated as online behavior, before our study we did not think these patterns were obvious in online behavior either (and scanning industry white papers, one will see that many others still do not find it obvious!). We believe the importance of activity bias in the offline domain is an open question. It is not difficult to come up with examples in which offline advertising exposure could spuriously correlate with dependent variables of interest. Billboards undoubtedly "cause" car accidents. Ads near hospitals "cause" illness. Restaurant ads near malls probably "cause" food consumption in general. Exposure to ads in the supermarket "saver" are likely correlated with consumption of unadvertised products. And so forth. The superior quality of data (and experiments) available in online advertising has laid bare the presence of activity bias in this domain. We believe the level of activity bias in other domains is an interesting, open question.

\section{Measuring the long-run returns to advertising}

Any study of advertising effectiveness invariably has to specify the window of time to be included in the study. While effects of advertising could in principle last a long time, in practice one must

\footnotetext{
${ }^{20}$ In some cases, even such placebo tests may fail as the qualifications for seeing the ad may be intrinsically correlated with the desired outcome as may be the case for remarketing and other forms of targeting which account for search activity and browsing behavior.
} 
pick a cut-off date. From a business perspective, making decisions quickly is an asset worth trading decision accuracy for at the margin. But can patient scholars (or firms) hope to measure the longrun effects of advertising? Here we address the statistical challenges of this question. The answer, unfortunately, is rather negative. As one moves further and further from the campaign date, the cumulative magnitude of the sales impact tends to increase. (This is not guaranteed, as ads could simply shift purchases forward in time, so a short time window could measure a positive effect while a long time window gives a zero effect. But in practice, we have so far noticed point estimates of cumulative effects to be increasing in the time window we have studied.) However, the amount of noise in the estimate tends to increase faster than the increase in the signal (treatment effect) itself because in the additional data the control and treatment groups look increasingly similar, making long-run studies less statistically feasible than short-run ones. In the remainder of this section we formalize and calibrate this argument.

We again employ the treatment vs. control $t$-statistic indexed by little $t$ for time. For concreteness, let time be denominated in weeks. For notational simplicity, we will assume constant variance in the outcome over time, no covariance in outcomes over time, ${ }^{21}$ constant variance across exposed and unexposed groups, and balanced group sizes. We will consider the long-term effects by examining a cumulative $t$-statistic (against the null of no effect) for $T$ weeks rather than a separate statistic for each week. We write the cumulative $t$-statistic for $T$ weeks as:

$$
t_{\Delta \bar{y}_{T}}=\sqrt{\frac{N}{2}}\left(\frac{\sum_{t=1}^{T} \Delta \bar{y}_{t}}{\sqrt{T} \hat{\sigma}}\right) .
$$

At first glance, this $t$-statistic appears to be a typical $O(\sqrt{T})$ asymptotic rate with the numerator being a sum over $T$ ad effects and the denominator growing at a $\sqrt{T}$ rate. This is where economics comes to bear. Since $\Delta \bar{y}_{t}$ represents the impact of a given advertising campaign during and following the campaign (since $t=1$ indexes the first week of the campaign), $\Delta \bar{y}_{t} \geq 0$. But the effect of the ad each week cannot be a constant-if it were, the effect of the campaign would be infinite. Thus, it is generally modeled to be decreasing over time.

With a decreasing ad effect, we should still be able to use all of the extra data we gather following the campaign to obtain more statistically significant effects, right? Wrong. Consider the condition necessary for an additional week to increase the $t$-statistic:

$$
\begin{aligned}
t_{\Delta \bar{y}_{T}} & <t_{\Delta \bar{y}_{T+1}} \\
\frac{\sum_{t=1}^{T} \Delta \bar{y}_{t}}{\sqrt{T}} & <\frac{\sum_{t=1}^{T+1} \Delta \bar{y}_{t}}{\sqrt{T+1}}
\end{aligned}
$$

\footnotetext{
${ }^{21}$ This assumption is clearly false: individual heterogeneity and habitual purchase behavior result in serial correlation in purchasing behavior. However, as we are considering the analysis over time, if we assume a panel structure with fixed effect or other residual-variance absorbing techniques to account for the source of this heterogeneity, this assumption should not be a first-order concern.
} 
Some additional algebra leads us to

$$
1+\frac{1}{T}<\left(1+\frac{\Delta \bar{y}_{T+1}}{\sum_{t=1}^{T} \Delta \bar{y}_{t}}\right)^{2}
$$

which approximately implies

$$
\frac{1}{2} \cdot \frac{1}{T} \sum_{t=1}^{T} \Delta \bar{y}_{t}<\Delta \bar{y}_{T+1} .
$$

This last expression says, "If the next week's expected effect is less than one-half the average effect over all previous weeks, then adding it in will only reduce precision." Thus, the marginal week can easily cloud the previous weeks, as its signal-to-noise ratio is not sufficiently large enough to warrant its inclusion. ${ }^{22}$ If the expected impact of the campaign following exposure decays rapidly (although not necessarily all the way to zero), it is likely that including additional weeks beyond the campaign weeks will decrease the statistical precision.

Suppose that you were just content with the lower bound of the confidence interval increasing in expectation. A similar calculation, under similar assumptions, shows that the lower bound of a $95 \%$ confidence interval will increase if and only if

$$
1.96(\sqrt{T+1}-\sqrt{T})<\frac{\Delta \bar{y}_{T+1}}{\hat{\sigma} / \sqrt{N}}
$$

where the right-hand expression is the marginal expected $t$-statistic of the $T+1^{\text {th }}$ week.

We can summarize these insights by returning to our formula for the $t$-statistic:

$$
t_{\Delta \bar{y}_{T}}=\sqrt{\frac{N}{2}}\left(\frac{\sum_{t=1}^{T} \Delta \bar{y}_{t}}{\sqrt{T} \hat{\sigma}}\right) .
$$

Since the denominator is growing at $O(\sqrt{T})$, in order for the $t$-statistic to grow, the numerator must grow at a faster rate. In the limit we know this cannot be as the the total impact of the advertising would diverge faster than even the harmonic series. ${ }^{23}$

Now ex-ante it is hard to know when the trade-off turns against you. The effect may decay slower than the harmonic series initially and then move towards zero quite quickly. Of course if we knew the pattern of decay, we would have answered the question the whole exercise is asking! So in the end the practitioner must make a judgment call. While choosing longer time frames for

\footnotetext{
${ }^{22}$ Note that this expression is completely general for independent random draws under any marginal indexing or ordering. In the identically distributed case, though, the expected mean for the marginal draw is equal to all inframarginal draws, so the inequality always holds.

${ }^{23}$ We note that an asset with infinite (nominal) returns is not implausible per se (a consol does this), but we do find infinite effects of advertising implausible. The harmonic series is $\sum \frac{1}{t}$ whereas the requisite series for an increasing $t$-statistic would be $\approx \sum \frac{1}{\sqrt{t}}$ which diverges much more quickly.
} 
advertising effectiveness analyses should capture more of the cumulative effect (assuming that it is generally positive), including additional weeks may just cloud the picture by adding more noise than ad impact. Measuring the effects of advertising inherently involves this sort of "judgment call" —an unsatisfying step in the estimation process for any empirical scientist. But the step is necessary since, as we have shown, estimating the long-run effect of advertising is a losing proposition-the noise eventually overwhelms the signal. The question is "when," and right now our judgment call is to use 1-4 weeks, but this is far from the final word.

\section{Advances in computational advertising}

In traditional media, targeting is typically a human-controlled process of determining the demographic groups most likely to consume the product. Readers may be familiar with Nielsen ratings for television, which break down to viewership by demographic categories. Campaigns often have "reach goals" for specific demographics a firm is interested in advertising to and marketing representatives use a portfolio of media outlets to meet these goals.

Online advertising opens up the possibilities for automated approaches to targeting because online ad delivery systems both gather information about specific users and make real-time adserving decisions. "Computational advertising" is described by one of the founders of the field, Andrei Broder, as "a principled way to find the best match between a given user in a given context and a suitable advertisement" (Broder, 2008). In traditional media, you have to specify who you want to advertise to. With computational advertising, you instead specify outcome metrics - an end-goal supported by the system - and automated systems determine how to achieve that goal most efficiently. The end goal could be online sign-ups, clicks to a sales page, and so on. A system will not support all end goals, and some supported goals, such as conversions, might exhibit slow learning because the success rate is so low (1 in 300,000 would not be uncommon for account sign-ups, for instance).

While the details of these systems are well beyond the scope of this paper, we'll give the flavor of how they work. Which display ad to show can be modeled as a multi-armed bandit problem. The possible ads are the "arms" and a user-ad pair is a "pull of the arm." Papers in this literature adapt classic machine learning tools to the ad-serving context (see for instance Pandey et al., 2007). Another approach, which is used in text-link based advertising, is to view the advertisement as a document that must be retrieved and matched to the page the ad is served on, which can be thought of as the query. As indicated by the terminology, this approach models ad-serving using the tools of web search (Rusmevichientong and Williamson, 2006; Cary et al., 2007).

We view (the current incarnation of) computational advertising primarily as automated targeting and local bid adjustment (to equalize CTR across campaigns, for instance). It helps locally optimize ad spend by minimizing costs for given a campaign goal. Using a high-level goal, such as clicks or sign-ups, combined with a budget, it reduces the need to set targeting dimensions (reduces 
not eliminates because one might still set priors for the learning system, which might matter a lot in slow-to-learn tasks) and funnels spend to better performing inventory. Focusing on high-level goals also helps shift the conversation from "who should get ads" to "what do we want to get from our ad spending." Practioners should be cautious, however, that the system does not conflate "the audience most likely to convert" to the "audience that delivers the most additional conversions." To see the difference, imagine a customer that would buy anyway, but finds it convenient to click on an ad if he sees one. Paying for this conversion is a total waste of money. In our experience, some automated systems fail to draw this distinction and in doing so, "order anticipate" by advertising to people likely to make a future purchase anyway. A natural solution is to integrate computational advertising with experimental platforms to provide randomization in order to measure incremental conversions.

Computational advertising is promising advance advertising science. It can improve efficiency in the market by providing a better match of advertisements to consumers, thereby creating value, but current systems do not solve all the challenges we have laid out thus far, such as how much of an given action should be attributed to a given ad. For instance, suppose an online brokerage calculates that it nets $\$ 100$ in profit from every account sign-up. Should it specify $\$ 100$ as a maximum bid an automated system and then "set it and forget it?" Well presumably the brokerage is advertising heavily on TV and other media, including other online media that was not the "last click." Bidding $\$ 100$ effectively says all this other spending gets zero credit — the firm would over-advertise using this rule. Of course this is just the "attribution problem" reframed from the advertiser's perspective.

The point can be stated succinctly: many of the difficulties we have discussed about globally optimizing ad spending apply to computational advertising as well. One could imagine a system that conducts automated experiments to measure incremental conversions and self-governs bids based on the experimental feedback, and so forth, but we are not there yet in practice. This is the type of advance that, if realized, would revolution the industry. We discuss this theme in the next section.

\section{Moving forward}

Digital measurement has opened up many doors in measuring advertising effectiveness, but many challenges persist. In this section we look toward the future and discuss how we think many of the existing challenges will be overcome. Overall we expect the advances to mainly come from better experimentation infrastructure to generate high-quality data at scale.

Perhaps the most important advance is the falling cost of experimentation. The first generation of field experiments we ran at Yahoo! randomly selected a relatively small sample of users targeted by the campaign to see an unrelated advertisement. The problem was that an unrelated ad had

to be entered into the booking system and run for the users that were randomized into the control group. The booking system was set up so that a firm could run multiple "creatives" (different 
versions of the ad) for fractions of traffic. What this meant was the unrelated ad had to be fully specified in the booking system. The firm for whom we ran the experiment did not want to let another retailer get the traffic, because the competitor would benefit from the targeting dimensions set up by the retailer (including, for instance, past purchasing behavior). The solution was to use charity ads for the control group. But this meant that either the advertiser had to pay for the control ads or Yahoo! had to donate them-both options came at a cost that increased linearly in the size of the control group, meaning that first generation experiments had relatively small control groups.

A small control group not only hurts power but also makes experimentation less useful as an evaluative tool. An experiment with $90 \%$ of subjects in the treatment group and $10 \%$ in the control has the same power as one with $10 \%$ in the treatment and $90 \%$ control. If control ads are free, then the an advertiser could run 9 of the latter for the cost of 1 of the former. ${ }^{24}$ For control ads to be free, the ad server needs to be able to serve the "next ad in line" every time a user is randomized into the control group. Technologically this requires a short serving latency between the request to the ad server, the randomization, and the request for the replacement ad. The replacement ads are known as "ghost ads" - ads that naturally qualified to be served to a given user targeted by the campaign under study but not associated with the advertiser. Ghost ads make exploration and evaluation cheaper. Small treatment groups limit cost and allow advertisers to hone copy early in a campaign, while free control subjects help evaluate the campaign ex-post.

Major online publishers are developing similar experimentation platforms. As experiments become cheaper and easier to run, advertisers will be able to form more precise beliefs on effectiveness than has heretofore been possible and further integrate experimentation into computational advertising platforms. These systems could incorporate an informative prior, which would help combat the power concerns we detailed earlier.

Another experimentation technology that improves power is the pre-experiment matching of users. To see how this works, consider an experiment with subjects spread across treatment and control 50-50. A standard experiment would simply flip a coin each time a user arrived at the website and show the ad corresponding to the outcome of the flip. Matching works as follows. Specify a set of attributes you care about such as recent sales levels and linear time trend. Form pairs of users by minimizing some objective function that defines the distance between two nodes in the graph of users. Then for each pair, flip a coin to determine experimental grouping. By construction the specified metrics should be almost exactly equal between the two groups. For evaluating a noisy variable such as sales, guaranteeing the pre-period sales were the same can be useful. The treatment assignment is still totally exogenous, so all our normal intuition on how experiments identify causal

\footnotetext{
${ }^{24}$ Note that the statistical gains from such a change in experimental design are 3-fold. Further altering the design, assuming constant returns to scale from advertising (Lewis, 2010; Johnson et al., 2012), by concentrating the $90 \%$ treatment group's ad impressions all within a smaller $10 \%$ treatment group expects an impact that is 9 times as large, resulting in the equivalent ad effectiveness insights from running 81 of the $90 \% / 10 \%$ experiments, producing confidence intervals of the ROI that are 9 times more precise at no additional advertising cost.
} 
effects goes through. Recent work has demonstrated that these techniques can double the power of experiments in many relevant settings (Deng et al., 2013).

The future is also looking up for evaluating television advertising and associated "cross media" interaction effects. More people are viewing TV through devices like the Xbox and through services like Google TV, both of which link users to ads in systems similar to major web publishers. Furthermore, these users often have identifiers that can link television, sponsored search, and display ads for a single individual. Never before in the history of advertising has this been possible. The ability to measure cross-channel effects with the reliability of randomized experiments opens the door to many new questions for academics and many new strategies for advertisers. As more forms of advertising become measurable on an individual level, our ability to provide reliable estimates of advertising effectiveness will expand as well. The advances so far have already set a new state-of-the-art in measurement, and we expect the trend to continue.

\section{Concluding remarks}

The science of measuring advertising effectiveness has evolved considerably due to new digital data sources and experimentation platforms. We view experimentation on the individual level with the ad delivery linked to purchasing behavior as a true game-changer offered by digital media as compared to traditional counterparts. Whether in search or display, new advertisers can gather feedback that is immune from the biases that plague observational methods. Another important advance is computational advertising. Computational advertising helps solve the targeting problem and usefully shifts the conversation from "who to hit" to "what do I get." Yet neither of these advances solve all the measurement problems in advertising science. Experiments are noisy and computational advertising still relies on humans to enter the key parameters, such as valuations of clicks or conversions, that govern spend.

Moving forward, experimentation and data collection technology is evolving alongside new forms of ad-serving and computational advertising systems. Questions such as the cross-derivative of certain media on the effectiveness of other media will be in play in the coming years. Measuring the

effectiveness of media, such as television, that were previously not technologically feasible, because randomizing delivery was not possible at scale, will also greatly expand knowledge on advertising effectiveness. This will in turn allow firms to more accurately guide their advertising expenditure. Our view, however, is that challenges such as the measuring long-run effects of advertising and the impact of "brand advertising" appear to be out of reach for at least the next 5-10 years, if not longer. We await new developments in advertising science at the digital frontier to facilitate the answers to these and new questions. 


\section{References}

Abraham, M. (2008). The off-line impact of online ads. Harvard Business Review, 86(4):28.

Bagwell, K. (2008). The economic analysis of advertising. Handbook of Industrial Organization, 3.

Blake, T., Nosko, C., and Tadelis, S. (2013). Consumer heterogeneity and paid search effectiveness: A large scale field experiment. NBER Working Paper, pages 1-26.

BLS (2010). In American Time Use Survey, volume http://www.bls.gov/tus/charts/leisure.htm.

Broder, A. (2008). Computational advertising and recommender systems. In Proceedings of the 2008 ACM conference on Recommender systems, pages 1-2. ACM.

Card, D. (1999). The causal effect of education on earnings. Handbook of labor economics, 3:18011863.

Cary, M., Das, A., Edelman, B., Giotis, I., Heimerl, K., Karlin, A., Mathieu, C., and Schwarz, M. (2007). Greedy bidding strategies for keyword auctions. In Proceedings of the 8th ACM conference on Electronic commerce, pages 262-271. ACM.

Chan, D., Ge, R., Gershony, O., Hesterberg, T., and Lambert, D. (2010). Evaluating online ad campaigns in a pipeline: causal models at scale. In Proceedings of the 16th ACM SIGKDD international conference on Knowledge discovery and data mining, pages 7-16. ACM.

Deng, A., Xu, Y., Kohavi, R., and Walker, T. (2013). Improving the sensitivity of online controlled experiments by utilizing pre-experiment data. In Proceedings of the sixth ACM international conference on Web search and data mining, pages 123-132. ACM.

Dickie, M., Fisher, A., and Gerking, S. (1987). Market transactions and hypothetical demand data: A comparative study. Journal of the American Statistical Association, pages 69-75.

Goldfarb, A. and Tucker, C. (2011a). Online display advertising: Targeting and obtrusiveness. Marketing Science, 30(3):389-404.

Goldfarb, A. and Tucker, C. E. (2011b). Privacy regulation and online advertising. Management Science, 57(1):57-71.

Gonen, R. and Pavlov, E. (2007). An incentive-compatible multi-armed bandit mechanism. In Proceedings of the twenty-sixth annual ACM symposium on Principles of distributed computing, pages 362-363. ACM.

Hu, Y., Lodish, L. M., and Krieger, A. M. (2007). An analysis of real world tv advertising tests: A 15-year update. Journal of Advertising Research, 47(3):341. 
Johnson, G., Lewis, R. A., and Reiley, D. H. (2012). Location, location, location: Geo-targeting increases effectiveness of online display advertising. Unpublished manuscript.

Johnson, G. H., Lewis, R. A., and Reiley, D. H. (2013). Add more ads? experimentally measuring incremental purchases due to increased frequency of online display advertising. In Working paper.

Kumar, D. and Yildiz, T. (2011). Measuring online ad effectiveness. In 12th ACM Conference on Electronic Commerce. ACM.

Lambrecht, A. and Tucker, C. (2011). When does retargeting work? information specificity in online advertising. SSRN Working Paper.

Lewis, R. (2010). Where's the "Wear-Out?": Online Display Ads and the Impact of Frequency. $\mathrm{PhD}$ thesis, MIT PhD Dissertation.

Lewis, R., Rao, J., and Reiley, D. (2011). Here, there, and everywhere: correlated online behaviors can lead to overestimates of the effects of advertising. In Proceedings of the 20th international conference on World wide web, pages 157-166. ACM.

Lewis, R. and Reiley, D. (2013a). Super bowl advertising causes down-to-the-minute online search behavior. In Proceedings of the 14th ACM Conference on Electronic Commerce. ACM.

Lewis, R., Reiley, D., and Schreiner, T. (2012). Ad attributes and attribution: Large-scale field experiments measure online customer acquisition. Unpublished manuscript.

Lewis, R. A. and Nguyen, D. T. (2013). A samsung ad and the ipad: Display advertising's spillovers to online search. Unpublished manuscript.

Lewis, R. A. and Rao, J. M. (2013). On the near-impossibility of measuring the returns to advertising. Unpublished manuscript.

Lewis, R. A. and Reiley, D. H. (2012). Advertising effectively influences older users: A yahoo! experiment measuring retail sales. Review of Industrial Organization, (forthcoming).

Lewis, R. A. and Reiley, D. H. (2013b). Online advertising and offline sales: Measuring the effects of retail advertising via a controlled experiment on yahoo! Unpublished manuscript.

Lodish, L., Abraham, M., Kalmenson, S., Livelsberger, J., Lubetkin, B., Richardson, B., and Stevens, M. (1995). How tv advertising works: A meta-analysis of 389 real world split cable tv advertising experiments. Journal of Marketing Research, 32(2):125-139.

Lovell, M. (2008). A simple proof of the fwl theorem. The Journal of Economic Education, $39(1): 88-91$. 
Mincer, J. (1962). On-the-job training: Costs, returns, and some implications. The Journal of Political Economy, 70(5):50-79.

Murphy, J., Allen, P., Stevens, T., and Weatherhead, D. (2005). A meta-analysis of hypothetical bias in stated preference valuation. Environmental and Resource Economics, 30(3):313-325.

Pandey, S., Chakrabarti, D., and Agarwal, D. (2007). Multi-armed bandit problems with dependent arms. In Proceedings of the 24th International Conference on Machine learning, pages 721-728. ACM.

Pandey, S. and Olston, C. (2006). Handling advertisements of unknown quality in search advertising. In Advances in Neural Information Processing Systems, pages 1065-1072.

Rusmevichientong, P. and Williamson, D. (2006). An adaptive algorithm for selecting profitable keywords for search-based advertising services. In Proceedings of the 7th ACM Conference on Electronic commerce, pages 260-269. ACM.

Tucker, C. E. (2012). The economics of advertising and privacy. International Journal of Industrial Organization, 30(3):326-329. 